\title{
Gemeinsam auf dem Weg zur Qualität
}

\section{Adrian Ritter}

Freier Journalist

\author{
In einer neuen Publikation diskutieren der Hausarzt Bruno Kissling und die Ethno- \\ login Andrea Abraham die Qualität in der Medizin am Beispiel der Hausarztmedi- \\ zin. Sie sind überzeugt: Qualität lässt sich nicht sichern, sondern nur bewusst \\ machen und entwickeln. Die SÄZ hat Bruno Kissling zum Gespräch getroffen.
}

Die Atmosphäre in der Praxis ist nur in Ausnahmefällen hektisch, der Hausarzt hört dem Patienten aufmerksam zu und erklärt ihm, warum er was macht. Am Schluss der Konsultation haben Arzt und Patient gemeinsam abgemacht, wie die Behandlung weitergeht und der Patient kann das Besprochene nachvollziehen: «Wer seinen Besuch beim Hausarzt etwa so erlebt, kann davon ausgehen: Hier ist ein Arzt um Qualität bemüht», sagt Bruno Kissling. Er sitzt am Arbeitstisch seiner Hausarztpraxis in einem Wohnquartier der Stadt Bern. Seit 1982 ist er hier als Hausarzt tätig. Und tagtäglich bemüht er sich, die soeben beschriebenen Aspekte im Kontakt mit seinen Patientinnen und Patienten zu leben.

Seit Jahrzehnten treibt ihn das Thema um: Was ist Qualität in der Medizin? Kann man sie messen? Wie

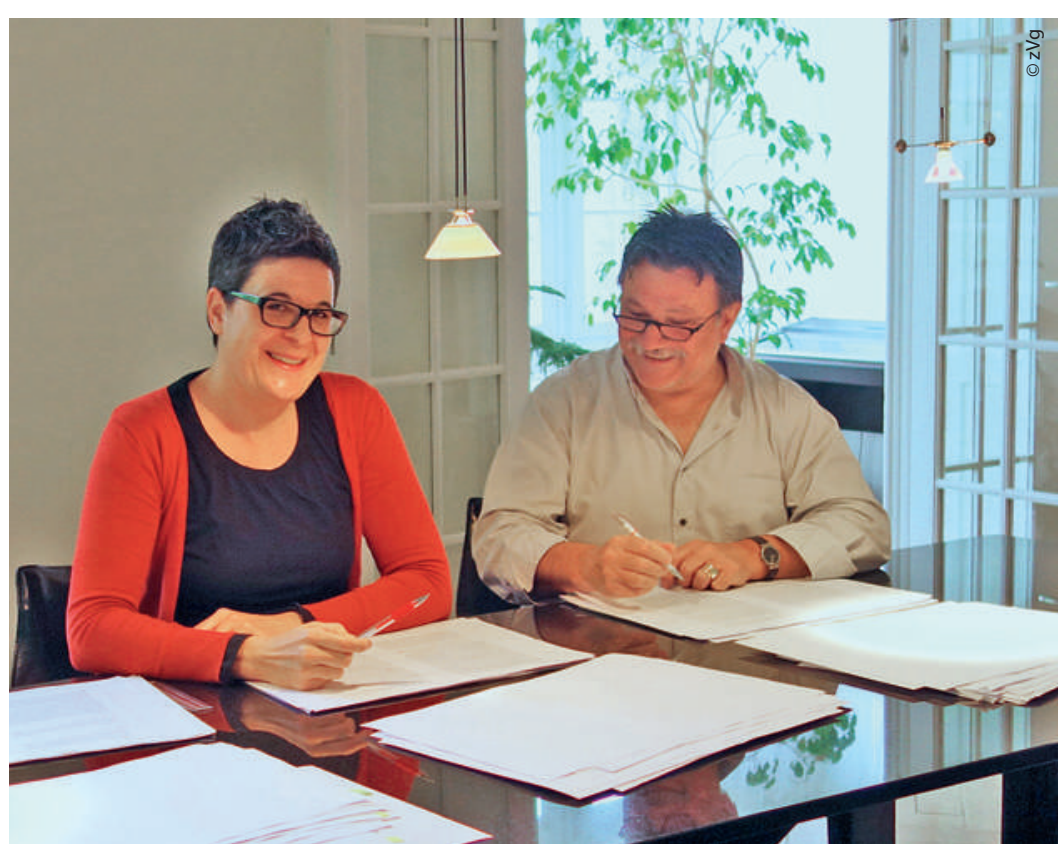

Auf dem Weg zum gemeinsamen Buch. Sein Thema: Die Geschichte des Qualitätsdiskurses und die vielfältigen Aspekte der Qualität - von der Ausbildung bis zur Fehlerkultur. soll sie gefördert werden? In der im Juni erschienenen Publikation Qualität in der Medizin. Briefe zwischen einem Hausarzt und einer Ethnologin legt er gemeinsam mit der Ethnologin Andrea Abraham seine Gedanken dazu dar (s. Box nächste Seite).

\section{Rund um die Uhr}

Zu Beginn seiner Tätigkeit als Hausarzt, beschreibt er im Buch, habe Qualität für ihn vor allem bedeutet: Rund um die Uhr für die Patienten da sein. «Spitalgläubig» habe er Untersuchungen durchgeführt und Behandlungen veranlasst, zu denen Spezialisten und Krankenhäuser ihn in ihren Berichten aufforderten.

Einem Burnout nahe und auf der Suche nach mehr Sinn in seiner Tätigkeit, begann er Kurse für systemischlösungsorientiertes Denken und Handeln zu besuchen und bildete sich in psychosomatischer und psychosozialer Medizin weiter. Seither sucht er nicht mehr Lösungen für, sondern mit seinen Patienten.

Seit 1995 bringt Bruno Kissling seine Vorstellung einer am Menschen orientierten Medizin auch standespolitisch ein. So war er u.a. im Vorstand der Schweizerischen Gesellschaft für Allgemeinmedizin, Chefredaktor der Hausärztezeitschrift PrimaryCare und Schweizer Delegierter beim Welthausärzteverband WONCA.

\section{Qualität im Zirkel}

Um mit Berufskolleginnen und Berufskollegen über die Qualität ihrer hausärztlichen Arbeit zu reflektieren, gründete Kissling 1998 den «Qualitätszirkel Elfenau» - benannt nach dem Quartier, in dem sich seine Praxis befindet. Es war eine der ersten solchen Gruppen in der Schweiz. Die Idee: Etwa zwölf Hausärztinnen und Hausärzte treffen sich circa monatlich zum Erfahrungsaustausch. 
Kissling war bis 2007 Moderator des Qualitätszirkels Elfenau. Nicht nur der Austausch über die Behandlung von Osteoporose oder über TARMED waren ihm ein Anliegen, sondern vor allem auch über die Komplexität und Qualität der Hausarztmedizin. Dominiert wurde diese Diskussion auf gesellschaftlicher Ebene seit den 1990er Jahren von der Evidenz-basierten Medizin (EBM) und der Forderung von Politikern und Krankenversicherern nach Standards der Qualitätssicherung und Guidelines. Wirksamkeit, Zweckmässigkeit und Wirtschaftlichkeit, so brachte das revidierte Krankenversicherungsgesetz 1996 auf eine Formel, was von den Ärzten erwartet wird.

\section{Komplexeste Medizin}

Für die Teilnehmenden im Qualitätszirkel um Bruno Kissling indes wurde immer klarer: Die Evidenz-basierte Medizin setzt zu stark auf Mittelwerte, bereinigt von der einzigartigen Situation jedes Patienten. Methoden der Qualitätsmessung, die auf die Hygiene im OP oder einen standardisierten chirurgischen Eingriff angebracht sein mögen, versagen in der Hausarztmedizin mit ihren oft chronischen und polymorbiden Krankheitsbildern.

«Die Hausarztmedizin ist die komplexeste aller medizinischen Disziplinen. Hier sind «soft facts» wie Kommunikation mit den Patienten für den Erfolg einer Behandlung oft entscheidend», sagt Kissling. Eine Behandlung, welche die Präferenzen des Patienten mit berücksichtigt, müsse bisweilen von Guidelines und Richtlinien abwei-

Das Buch von Andrea Abraham und Bruno Kissling:

\section{Qualität in der Medizin}

Briefe zwischen einem Hausarzt und einer Ethnologin. Basel: EMH Media Schweizerischer Ärzteverlag; 2015. 177 Seiten, 7 farbige Abbildungen. 19.50 CHF. ISBN 978-3-03754-084-8

In ihrem Gedankenaustausch erörtern der Hausarzt Bruno Kissling und die Ethnologin Andrea Abraham die vielfältigen Aspekte von Qualität in der Medizin. Bruno Kissling bringt seine 30-jährige Berufserfahrung als Hausarzt, Andrea Abraham die Ergebnisse ihrer qualitativen Erforschung der Hausarztmedizin ein. Die Autoren zeigen auf, wie die moderne Idee des Qualitätsmanagements nach dem Zweiten Weltkrieg in der US-Kriegswaffen- und der japanischen Automobilindustrie begann. Wo jedes produzierte Auto einem bestimmten Autotyp entsprechen muss, machen quantitative Methoden der Qualitätssicherung Sinn. Bei Patientinnen und Patienten hingegen sei Varianz die Norm. Qualität sei in einem solchen Kontext etwas Unfertiges, das sich nicht sichern, sondern nur entwickeln lasse. Die Publikation ist in Dialogform verfasst. Sie richtet sich in allgemeinverständlicher Sprache und mit zahlreichen Fallgeschichten sowohl an medizinische Fachpersonen als auch an ein interessiertes nicht-medizinisches Publikum. Eine lohnenswerte Lektüre, welche die aktuelle Debatte über die Qualität in der Medizin anschaulich und spannend abbildet und reflektiert.

\section{Buchvernissage und Lesung}

Im Rahmen der SwissFamilyDocs Conference (27./28. August 2015, BERNEXPO, Bern) lädt EMH Media Schweizerischer Ärzteverlag zur Vernissage des Buches. Signierstunde mit den Autoren: Donnerstag, 27. August 2015, 11.45-13.15 Uhr, am Stand von EMH und FMH (Halle 1.2.). Lesung: Freitag, 28.8.2015, 12.45-13.30 Uhr, Special Focus Session 2 (Der Raum wird im Kongressprogramm bekanntgegeben).

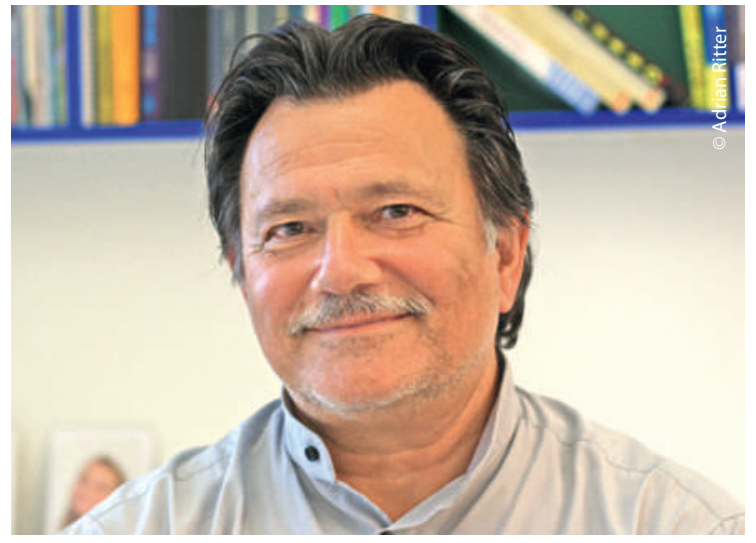

Bereits im Jahr 1998 gründete Bruno Kissling den «Qualitätszirkel Elfenau», schweizweit war es einer der ersten.

chen. Und die Qualität einer solchen personenzentrierten Medizin lässt sich nicht mit quantitativen Methoden messen. Es brauche qualitative Methoden, wie sie in der Sozialforschung genutzt werden.

So entwickelten sich in den 1990er Jahren denn auch die narrativ-basierte Medizin, die Komplexitätstheorie und systemisch-lösungsorientierte Ansätze als kritischer Gegendiskurs zur Evidenz-basierten Medizin und zur Ökonomisierung, Technisierung und Spezialisierung der Medizin.

\section{Qualität als Argument}

Der Qualitätszirkel Elfenau verfolgte die Diskussion mit Interesse und wurde selber zum Akteur, suchte den Kontakt zur Berner Ethnologin Andrea Abraham. Diese widmete in der Folge ihre Dissertation der Erforschung des Qualitätsdiskurses in der Medizin. Sie nahm unter anderem an Sitzungen des Qualitätszirkels teil und führte Gespräche mit Hausärztinnen und Hausärzten.

Das Fazit ihrer Arbeit: Es gibt keine objektiven Kriterien der Qualitätsmessung. Allen Kriterien liegen Erkenntnisse, Annahmen und Interessen zugrunde. Insbesondere seit der Revision des Krankenversicherungsgesetzes wurde der Begriff der Qualität zu einem obligaten Bestandteil des politischen Argumentariums. Auch die Hausärzte setzten die Qualitätsrhetorik zunehmend bewusst ein - um für Investitionen in die Hausarztmedizin zu werben, beschrieb Abraham.

\section{Entwicklung von unten}

Damit die Dissertation nicht in der Schublade verstaubte, lud Andrea Abraham Bruno Kissling als KoAutor ein, die Ergebnisse und ihre beiden Sichtweisen in Dialogform zu veröffentlichen. In der gemeinsamen Publikation beleuchten sie sowohl die Geschichte des 


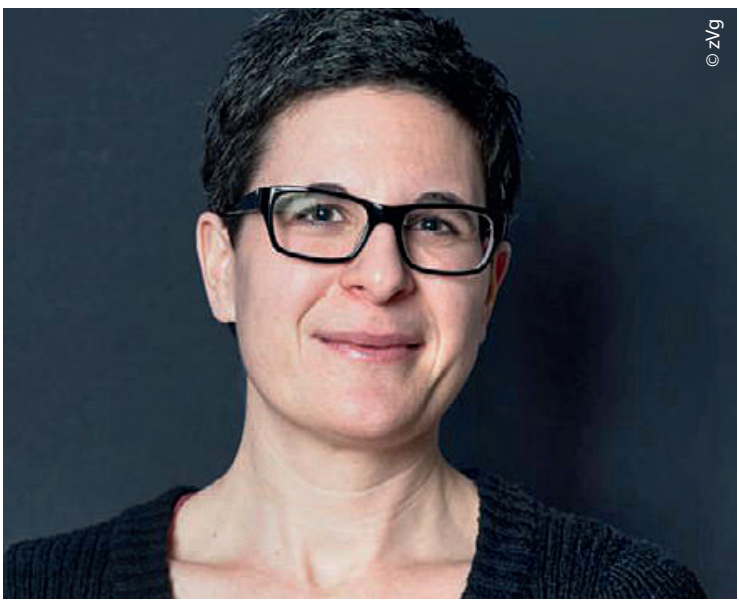

Widmete ihre Dissertation der Erforschung des Qualitätsdiskurses in der Medizin: die Ethnologin Andrea Abraham.

Qualitätsdiskurses wie auch die vielfältigen Aspekte von Qualität in der Medizin - von der Ausbildung bis zur Fehlerkultur.

Sie vertreten dabei dezidiert die Ansicht: Gute Entscheidungsprozesse von Ärztinnen und Ärzten berücksichtigen einerseits selbstverständlich epidemiologische und biomedizinische Aspekte, beziehen andererseits aber auch Erfahrung, Intuition, ethische Überlegungen und die Wünsche und individuelle Situation der Patientinnen und Patienten mit ein.

Abraham und Kissling sind überzeugt: Die Qualität in der Medizin kann nicht gesichert werden. Sie kann nur bewusst gemacht, gefördert und entwickelt werden. Es ist eine Sichtweise, die weit weg ist von den Vorschlägen, die der Bundesrat im Mai 2015 präsentierte und forderte: Qualität in der Medizin muss besser gemessen und der Einsatz anerkannter Standards verbindlich geregelt werden. Von qualitativen Methoden war nirgends die Rede. "Guidelines, die der Komplexität in der Hausarztmedizin gerecht werden, gibt es bisher nicht. Und es gibt in einem interaktiven Prozess auch keine Sicherungsmethode, um Fehler garantiert zu vermeiden", sagt Bruno Kissling. Hohe Qualität müsse trotzdem immer die Ausrichtung und das Ziel des hausärztlichen Handelns sein.

Die Beachtung der so wichtigen "soft facts» könne erlernt werden. Die Qualitätsentwicklung müsse daher

\section{Am Puls der Hausärzte}

Im Rahmen des Qualitätszirkels Elfenau entstand die Idee, eine Dokumentarfilm-Trilogie über Hausärzte zu realisieren. Die Idee wurde von der Filmemacherin Sylviane Gindrat umgesetzt und hatte 2013 Premiere. In drei Filmen begleitet sie vier Hausärzte und zwei Hausärztinnen in ihrem Alltag in der Stadt, auf dem Land und in einem Bergtal. Die Filmemacherin war selber 20 Jahre lang Hausärztin. Die Filme sollen jetzt um ein Lehrmittel ergänzt zur Schulung angehender Ärztinnen und von der Basis her geschehen: In der Aus- und Weiterbildung gelte es, Kommunikation und systemischlösungsorientiertes Handeln vermehrt zu vermitteln. Als besonders hilfreich erachtet Kissling zudem Supervisionen und Qualitätszirkel - letztere sind für Mitglieder von Hausarzt-Netzwerken heute obligatorisch.

\section{Geteilte Verantwortung}

Gefragt ist aber nicht nur das Engagement der Ärztinnen und Ärzte. Die Rolle der Patienten als «Ko-Produzenten von Qualität» und ihre Mitverantwortung für die steigenden Gesundheitskosten werden zu wenig thematisiert, stellen Abraham und Kissling fest. Sehr oft würden Patienten nach allen technisch machbaren Behandlungen verlangen - ungeachtet der Kosten. Für Kissling liegt die Qualität für den Patienten aber oft auch im Weglassen von Untersuchungen und Therapien.

Er sieht auch hier die Kommunikationskunst des Arztes gefragt: «Die Regel sollte sein, dass Arzt und Patient gemeinsam eine Behandlung von hoher Qualität und zu verantwortlichen Kosten beschliessen. Wo ein solches 'Shared Decision Making' zustande kommt, bleiben meist auch die Kosten in einem vernünftigen Rahmen", so seine Erfahrung.

\section{Hausarztmedizin stärken}

Um die Kosten für das Gesundheitswesen in den Griff zu bekommen, seien auch strukturelle Veränderungen nötig - zu gross sei heute die Zahl der Spezialisten im Vergleich zu den Allgemeinmedizinern. Es gelte, die medizinische Grundversorgung durch Hausärzte zu stärken. Kissling sieht dabei Lichtstreifen am Horizont: Junge Ärztinnen und Ärzte entschieden sich wieder öfter für diesen Berufsweg, nicht zuletzt dank der Bemühungen des Verbandes «Junge Hausärztinnen und -ärzte Schweiz». Neue Notfallmodelle in Zusammenarbeit mit den Spitälern würden die Hausärzte von der Dienstpflicht in der Nacht und am Wochenende entlasten, was den Beruf attraktiver mache. So würden auch ältere Hausärzte bisweilen über die Pensionierung hinaus arbeiten.

Auch bei Bruno Kissling - 66-jährig - steht die Nachfolgeregelung an. Ein Hausarzt im selben Quartier ist dabei mit einer guten Lösung an ihn herangetreten. Sie legen ihre Praxen zusammen und übergeben sie einem jungen Kollegen. Bis 2018 wird Kissling noch 50 Prozent dort tätig sein. Und jeweils am Ende einer Konsultation seine Patienten fragen: «Stimmt es so für Sie? War die Konsultation so, wie sie es sich vorgestellt haben?» Qualität als Kommunikation. Kommunikation als Qualität. 